\title{
COVARIANCES OF GENERALIZED PROCESSES WITH ORTHOGONAL VALUES
}

\author{
LEWIS PAKUL A
}

ABSTRACT. A general form for the covariance of a generalized process with orthogonal values is found in the case where the covariance $B$ depends on test functions and their first derivatives. Specifically, if $B(\phi, \phi)=$ $\int \phi^{2} d \mu_{0}+\int \phi \phi^{\prime} d \mu_{1}+\int \phi^{2} d \mu_{2} \geq 0$ for $\phi \in \mathscr{D}(\mathbf{R})$ and Radon measures $\mu_{0}, \mu_{1}$, $\mu_{2}$, then there exist Radon measures $\nu_{0}, \nu_{1}, \nu_{2}$ such that $B(\phi, \phi)=$ $\int \phi^{2} d \nu_{0}+\int \phi \phi^{\prime} d \nu_{1}+\int \phi^{\prime 2} d \nu_{2}$ and, moreover, $\int f^{2} d \nu_{0}+\int f g d \nu_{1}+\int g^{2} d \nu_{2} \geq 0$ for all $f, g \in \mathscr{D}(\mathbf{R})$.

Let $X$ be a real second order generalized stochastic process with orthogonal values, that is $X: \mathscr{D}(\mathbf{R}) \rightarrow H$ is a continuous linear map where $\mathscr{D}(\mathbf{R})$ is the space of real infinitely differentiable functions with compact support having the usual inductive limit topology and $H$ is a Hilbert space (of square integrable random variables, say). Moreover, if $\phi, \psi$ have disjoint supports then $X(\phi)$ and $X(\psi)$ are orthogonal. Then the covariance $B(\phi, \psi)=\langle X(\phi)$, $X(\psi)\rangle_{H}$ is given by

$$
B(\phi, \psi)=\sum_{i, j=0}^{\infty} \int \phi^{(i)} \psi^{(j)} d \mu_{i j}, \quad B(\phi, \phi) \geq 0,
$$

where $\phi^{(i)}$ is the $i$ th derivative, and the $\mu_{i j}$ are Radon measures on $\mathbf{R}$ such that only finitely many of the measures $\left|\mu_{i j}\right|$ are nonzero on any bounded set. (See Gel'fand and Vilenkin [1964].) Another characterization of the se covariances has been given by Fernique [ 1967, Chapter IV]. We study in detail the case in which $B(\phi, \psi)$ depends only on $\phi, \psi$ and their first derivatives, i.e.,

$$
B(\phi, \psi)=\sum_{i, j=0}^{1} \int \phi^{(i)} \psi^{(j)} d \mu_{i j} .
$$

From the symmetry of $B$ we can assume $\mu_{01}=\mu_{10}$. Since $B$ is a (possibly degenerate) inner product, it is determined by the values

$$
B(\phi, \phi)=\int \phi^{2} d \mu_{0}+\int \phi \phi^{\prime} d \mu_{1}+\int \phi^{\prime 2} d \mu_{2} \geq 0
$$

where $\mu_{0}=\mu_{00}, \mu_{1}=\mu_{01}+\mu_{10}, \mu_{2}=\mu_{11}$. The representation (2) is not unique, in fact:

Proposition 1. $\int \phi^{2} d \mu_{0}+\int \phi \phi^{\prime} d \mu_{1}+\int \phi^{\prime 2} d \mu_{2}=0$ for all $\phi \in \mathscr{D}(\mathbf{R})$ if and only if $d \mu_{2}=0, d \mu_{1}=-2 h d x$ and $d \mu_{0}=d h$ for some function $h$ of bounded variation.

Received by the editors August 19, 1974.

AMS (MOS) subject classifications (1970). Primary 46F99, 60G20. 
Proof. Let $T$ and $S$ be the distributions given by

$$
T(\phi)=\int \phi d \mu_{0}+\frac{1}{2} \int \phi^{\prime} d \mu_{1} \text { and } S(\phi)=\int \phi d \mu_{2} .
$$

If $T\left(\phi^{2}\right)+S\left(\phi^{\prime 2}\right)=0$ for all $\phi$ then $T=0$. For let $f \in \mathcal{D}(\mathbf{R})$ have support contained in $[-c, c]$ and choose $g \in \mathscr{D}(\mathbf{R})$ such that $g(x)=1$ for $x \in[-c, c]$. Then $f=1 / 4\left[(f+g)^{2}-(f-g)^{2}\right]$ while $1 / 4\left[\left(f^{\prime}+g^{\prime}\right)^{2}-\left(f^{\prime}-g^{\prime}\right)^{2}\right]=f^{\prime} g^{\prime}=0$. It follows that $T(f)+S(0)=0$ so $T=0$. Now choose $g_{1}$ such that $g_{1}^{\prime}=1$ on $[-c, c]$. Then $f^{\prime}=1 / 4\left[\left(f^{\prime}+g_{1}^{\prime}\right)^{2}-\left(f^{\prime}-g_{1}^{\prime}\right)^{2}\right]$ and we deduce that $S\left(f^{\prime}\right)=0$, so $S$ is some multiple of Lebesgue measure which must clearly be 0 . Thus $\mu_{2}=0$; the fact that $T=0$ implies that $d \mu_{0}=d \cdot h$ for some $h$ of bounded variation. The converse follows by integration by parts. Q.E.D.

Let $\mathscr{D}^{0}(\mathbf{R})$ [resp., $\mathfrak{D}^{1}(\mathbf{R})$ ] be the space of continuous real-valued functions on $\mathbf{R}$ with compact support [resp., continuously differentiable functions with compact support] and denote the triplet $\left\langle\mu_{0}, \mu_{1}, \mu_{2}\right\rangle$ of Radon measures by $\mu_{\text {. }}$ Define quadratic forms

$$
Q_{\mu}(\phi)=\int \phi^{2} d \mu_{0}+\int \phi \phi^{\prime} d \mu_{1}+\int \phi^{\prime 2} d \mu_{2} \text { on } \mathfrak{D}^{1}(\mathbf{R})
$$

and

$$
\bar{Q}_{\mu}(\langle f, g\rangle)=\int f^{2} d \mu_{0}+\int f g d \mu_{1}+\int g^{2} d \mu_{2} \text { on } \mathscr{D}^{0}(\mathbf{R}) \times \mathscr{D}^{0}(\mathbf{R}) .
$$

Example. Let $\mu=\left\langle 2 \delta_{0}-\delta_{1}, 0,2 I_{[0,1]} d x\right\rangle$ where $\delta_{a}$ is the point mass at $a$ and $I_{[0,1]}$ is the indicator function on $[0,1]$. Then $Q_{\mu}$ is nonnegative definite, i.e., $Q_{\mu}(\phi) \geq 0$ for $\phi \in \mathfrak{D}^{1}(\mathbf{R})$, but $\bar{Q}_{\mu}$ is not nonnegative definite on $\mathscr{D}^{0}(\mathbf{R}) \times \mathfrak{D}^{0}(\mathbf{R})$. However if we let

$$
\nu=\left\langle 2(1+x)^{-2} I_{[0,1]} d x, 4(1+x)^{-1} I_{[0,1]} d x, 2 I_{[0,1]} d x\right\rangle,
$$

then $Q_{\mu}(\phi)=Q_{\nu}(\phi)$ for all $\phi \in \mathscr{D}^{1}(\mathbf{R})$ while $\bar{Q}_{\nu}(\langle f, g\rangle) \geq 0$ for $\langle f, g\rangle \in \mathfrak{D}^{0} \times$ $\mathfrak{D}^{0}$.

The following theorem asserts that a quadratic form $Q_{\mu}$ of the form (3) can always be (assumed to be) given by a triplet of measures $\mu$ such that $\bar{Q}_{\mu}$ is nonnegative definite on $\mathfrak{D}^{0} \times \mathfrak{D}^{0}$.

Theorem 1. If $\mu$ is a triplet of Radon measures on $\mathbf{R}$ such that $Q_{\mu}(\phi) \geq 0$ for all $\phi \in \mathfrak{D}^{1}$, then there exists a triplet $\nu$ such that

(a) $Q_{\mu}(\phi)=Q_{\nu}(\phi)$ for $\phi \in \mathfrak{D}^{1}$,

(b) $\bar{Q}_{\nu}(\langle f, g\rangle) \geq 0$ for all $f, g \in \mathfrak{D}^{0} \times \mathscr{D}^{0}$.

Lemma. If $Q_{\mu}$ is nonnegative definite, then $\mu_{2}$ is a nonnegative Radon measure.

Proof. Let $h$ be a function of bounded variation such that $\mu_{0}=d h$. Then integrating by parts we have $\int \phi^{2} d \mu_{0}+\int \phi \phi^{\prime} d \mu_{1}=\int \phi \phi^{\prime}\left(d \mu_{1}-2 h d x\right)$. Thus we can assume that $Q_{\mu}$ is given by (3) with $\mu_{0}=0$. If $\mu_{2}$ is not nonnegative, there exists a compact set $K \subset \mathbf{R}$. such that $\mu_{2}(K)=-\epsilon, \epsilon>0$. Let $U$ be an 
open relatively compact set, $U \supset K$, such that $\left|\mu_{2}\right|(U \backslash K)<\epsilon / 10$ where $\left|\mu_{2}\right|$ is the total variation measure of $\mu_{2}$. Let $\psi \in \mathscr{D}^{1}(\mathbf{R})$ be such that $0 \leq \psi \leq 1$, $\psi(x)=1$ for $x \in K, \psi(x)=0$ for $x \notin U$. Let

so that

$$
\phi_{s, n}(x)=\psi(x)(\sin n x) / n, \quad \phi_{c, n}(x)=\psi(x)(\cos n x) / n
$$

$$
\phi_{s, n}^{\prime}(x)^{2}=\psi(x)^{2} \cos ^{2} n x+f_{s, n}(x) ; \quad \phi_{c, n}^{\prime}(x)^{2}=\psi(x)^{2} \sin ^{2} n x+f_{c, n}(x)
$$

and $\left\|f_{s, n}\right\|_{\infty},\left\|f_{c, n}\right\|_{\infty},\left\|\phi_{s, n} \phi_{s, n}^{\prime}\right\|_{\infty},\left\|\phi_{c, n} \phi_{c, n}^{\prime}\right\|_{\infty}$ all approach 0 as $n \rightarrow \infty$. Let $N$ be so large that

$$
\left\|\phi_{s, N}^{\prime} \phi_{s, N}\right\| \leq \epsilon / 10\left|\mu_{1}\right|(U), \quad\left\|\phi_{c, N}^{\prime} \phi_{c, N}\right\| \leq \epsilon / 10\left|\mu_{1}\right|(U),
$$

Then

$$
\left\|f_{s, N}\right\| \leq \min \left(\epsilon / 10\left|\mu_{2}\right|(K), 1\right), \quad\left\|f_{c, N}\right\| \leq \min \left(\epsilon / 10\left|\mu_{2}\right|(K), 1\right) .
$$

$$
\int_{K} \psi(x)^{2} \cos ^{2} N x d \mu_{2}+\int_{K} \psi(x)^{2} \sin ^{2} N x d \mu_{2}=\mu_{2}(K)=-\epsilon .
$$

Thus one of the two integrals on the left side of (6) must be less than $-\epsilon / 2$. Suppose $\int_{K} \psi^{2} \sin ^{2} N x d \mu_{2}<-\epsilon / 2$. Then

$$
\begin{aligned}
& \int \phi_{c, N} \phi_{c, N}^{\prime} d \mu_{1}+\int \phi_{c, N}^{\prime 2} d \mu_{2} \\
& \quad=\int_{U} \phi_{c, N} \phi_{c, N}^{\prime} d \mu_{1}+\int_{U \backslash K} \phi_{c, N}^{\prime 2} d \mu_{2}+\int_{K} \phi_{c, N}^{\prime 2} d \mu_{2} .
\end{aligned}
$$

But from (5)

$$
\left|\int_{U} \phi_{c, N} \phi_{c, N}^{\prime} d \mu_{1}\right| \leq \frac{\epsilon}{10}, \quad\left|\int_{U \backslash K} \phi_{c, N}^{\prime 2} d \mu_{2}\right| \leq \frac{\epsilon}{5}
$$

and

$$
\int_{K} \phi_{c, N}^{\prime 2} d \mu_{2}=\int_{K} \psi^{2} \sin ^{2}(N x) d \mu_{2}+\int_{K} f_{c, N} d \mu_{2}<(\epsilon / 10)-(\epsilon / 2) .
$$

Thus $2_{\mu}\left(\phi_{c, N}\right)<0$. If the other integral in (6) were less than $-\epsilon / 2$ we could show $Q_{\mu}\left(\phi_{s, N}\right)<0$. In either case we have a contradiction so $\mu_{2}$ is nonnegative.

Proof of theorem. Let $\mathfrak{D}^{\circ}$ be given the usual inductive limit topology and let $V=\mathfrak{D}^{0} \oplus \mathfrak{D}^{0} \oplus \mathfrak{D}^{0}$. Define a subspace $M$ and a cone $K$ in $V$ as follows:

$$
\begin{gathered}
M=\left\{\left\langle\phi_{0}, \phi_{1}, \phi_{2}\right\rangle: \phi_{0} \in \mathfrak{L}^{1} \text { and } \phi_{1}=1 / 2 \phi_{0}^{\prime}\right\}, \\
K=\left\{\left\langle\phi_{0}, \phi_{1}, \phi_{2}\right\rangle: \phi_{0}(x) \geq 0, \phi_{2}(x) \geq 0, \phi_{0}(x) \phi_{2}(x)-\phi_{1}(x)^{2} \geq 0 \text { for all } x \in \mathbf{R}\right\} .
\end{gathered}
$$

Let

$$
\Phi\left(\left\langle\phi_{0}, \phi_{1}, \phi_{2}\right\rangle\right)=\int \phi_{0} d \mu_{0}+\int \phi_{1} d \mu_{1}+\int \phi_{2} d \mu_{2},
$$

so $\Phi$ is a continuous linear functional on $V$. We show that $\Phi$ is nonnegative on $M \cap K$. For $\left\langle\phi_{0}, \phi_{1}, \phi_{2}\right\rangle \in M \cap K$ let $c>0$ be such that supp $\phi_{0} \subset[-c, c]$, and let $a \in D^{1}$ be a nonnegative function such that $\alpha=1$ in a neighborhood of $[-c, c]$. Set 


$$
f_{n}(x)=\alpha(x)\left(\phi_{0}(x)+n^{-1}\right)^{1 / 2} .
$$

Since $\alpha^{\prime} \phi_{0}=\alpha^{\prime} \phi_{0}^{\prime}=0$, we get

$$
f_{n} f_{n}^{\prime}=n^{-1} \alpha \alpha^{\prime}+\phi_{1}, \quad f_{n}^{\prime 2}=n^{-1} \alpha^{\prime 2}+\alpha^{2} \phi_{1}^{2}\left(\phi_{0}+n^{-1}\right)^{-1},
$$

so $\lim _{n \rightarrow \infty}\left\|\phi_{0}-f_{n}^{2}\right\|_{\infty}=0, \lim _{n \rightarrow \infty}\left\|\phi_{1}-f_{n} f_{n}^{\prime}\right\|_{\infty}=0$. If $|x| \geq c, f_{n}^{\prime}(x)^{2}=$ $n^{-1} \alpha^{\prime}(x)^{2} \rightarrow 0$ as $n \rightarrow \infty$. If $|x|<c$ we must have $\phi_{2}(x) \geq f_{n}^{\prime}(x)^{2}$. For if $\phi_{2}(x)<f_{n}^{\prime}(x)^{2}$ we would have $\phi_{2}(x) \phi_{0}(x)-\phi_{1}(x)^{2}<-\phi_{2}(x) n^{-1}$ which is impossible by assumption. Thus $\lim _{n \rightarrow \infty}\left(\phi_{2}-f_{n}^{\prime 2}\right) \geq 0$.

Thus $Q_{\mu}\left(f_{n}\right) \geq 0$, the previous lemma, and Fatou's lemma imply that $\Phi\left(\left\langle\phi_{0}, \phi_{1}, \phi_{2}\right\rangle\right) \geq 0$.

The pair $(V, K)$ is a partially ordered topological vector space, and we have shown that $\Phi$ is a positive linear functional on the subspace $(M, M \cap K)$. We use the following results (see Namioka [1957]):

(I) If $M$ is a subspace of a partially ordered t.v.s. $(V, K)$ such that for each positive element $x \in V$ there exists $y \in M$ which dominates $x$ (i.e., $y-x \in K$ ), then each positive linear functional on $M$ can be extended to a positive linear functional on $V$.

(II) Let $(V, K)$ be a partially ordered LF space which can be defined by a nest of Fréchet spaces $\left\{V_{n}\right\}$ such that $K$ is closed in $V$ and $\left(V_{n} \cap K\right)-$ $\left(V_{n} \cap K\right)=V_{n}$ for all $n$. Then each positive linear functional is continuous.

$V, K, M$ satisfy the hypothesis of (I): Suppose $\left\langle\phi_{0}, \phi_{1}, \phi_{2}\right\rangle \in K$ and suppose $[-c, c]$ contains the supports of $\phi_{0}, \phi_{1}$ and $\phi_{2}$. Let $\alpha \in \mathfrak{D}^{1}$ be such that $a=\left(2\left\|\phi_{0}\right\|_{\infty}\right)^{1 / 2}$ on $[-c, c]$. Then $\left\langle\alpha^{2}, \alpha \alpha^{\prime}, \alpha^{\prime 2}+2 \phi_{2}\right\rangle \in M$ and dominates $\left\langle\phi_{0}, \phi_{1}, \phi_{2}\right\rangle$, i.e.,

since

$$
\left\langle\alpha^{2}-\phi_{0}, \alpha \alpha^{\prime}-\phi_{1},\left(\alpha^{\prime 2}+2 \phi_{2}\right)-\phi_{2}\right\rangle \in K,
$$

$$
\left(\alpha(x)^{2}-\phi_{0}(x)\right)\left(\alpha^{\prime}(x)^{2}+\phi_{2}(x)\right)-\left(\alpha(x) \alpha^{\prime}(x)-\phi_{1}(x)\right)^{2} \geq 0
$$

for all $x \in \mathrm{R}$ can be verified by considering $|x|<c$ and then $|x| \geq c$.

To show that the hypothesis of (II) is satisfied, note that $V$ is the inductive limit of the Fréchet spaces

where

$$
V_{n}=\mathfrak{D}^{0}[-n, n] \oplus \mathfrak{D}^{0}[-n, n] \oplus \mathfrak{D}^{0}[-n, n]
$$

$$
\mathfrak{D}^{0}[-n, n]=\left\{\phi \in \mathfrak{D}^{0}(\mathbf{R}): \operatorname{supp} \phi \subset[-n, n]\right\} \text {. }
$$

If $\phi_{0}, \phi_{1}, \phi_{2} \in V_{n}$, write $\phi_{0}=\phi_{0}^{+}-\phi_{0}^{-}, \phi_{2}=\phi_{2}^{+}-\phi_{2}^{-}$where $\phi_{0}^{+}=$ $\max \left(0, \phi_{0}\right)$ and $\phi_{0}^{-}=\max \left(0,-\phi_{0}\right)$. Let $\psi_{0}=|| \phi_{1}\left|-\phi_{0}^{+}\right|, \psi_{2}=|| \phi_{1}\left|-\phi_{2}^{+}\right|$. Then

$$
\left\langle\phi_{0}, \phi_{1}, \phi_{2}\right\rangle=\left\langle\psi_{0}+\phi_{0}^{+}, \phi_{1}, \psi_{2}+\phi_{2}^{+}\right\rangle-\left\langle\psi_{0}+\phi_{0}^{-}, 0, \psi_{2}+\phi_{2}^{-}\right\rangle .
$$

The second term on the right in (7) clearly belongs to $V_{n} \cap K$. To see that the first term belongs to $V_{n} \cap K$, note that $\psi_{0}+\phi_{0}^{+} \geq\left|\phi_{1}\right|, \psi_{2}+\phi_{2}^{+} \geq\left|\phi_{1}\right|$ and $\left\langle|\phi|_{1}, \phi_{1},\left|\phi_{1}\right|\right\rangle \in K$. Finally it is easy to see that $K$ is closed in $V$. 
Thus the functional $\Phi$ on $M$ extends to a continuous linear functional on $V$, that is, there are Radon measures $\left\langle\nu_{0}, \nu_{1}, \nu_{2}\right\rangle$ such that

$$
\Phi\left(\left\langle\phi_{0}, \phi_{1}, \phi_{2}\right\rangle\right)=\int \phi_{0} d \nu_{0}+\int \phi_{1} d \nu_{1}+\int \phi_{2} d \nu_{2}
$$

and $\Phi$ is nonnegative on $K$. But since $\left\langle f^{2}, f g, g^{2}\right\rangle \in K$ for all $f, g \in \mathscr{D}^{0}(\mathbf{R})$ we have conclusion (b) of the theorem. Q.E.D.

The triplet of measures $\left\langle\nu_{0}, \nu_{1}, \nu_{2}\right\rangle$ whose existence is guaranteed by Theorem 1 is not unique, although by Proposition 1 we must have $\nu_{2}=\mu_{2}$ for any such triplet. For example, the measure triplets $\langle 1+|x| d x,-2 x d x,|x| d x\rangle$ and $\langle|x| d x, 0,|x| d x\rangle$ define distinct positive linear functionals on $(V, K)$ which agree on $M$.

It can be shown that if a triplet $\nu$ satisfies conclusion (b) of Theorem 1 then:

(i) $\nu_{0} \geq 0, \nu_{2} \geq 0$;

(ii) $\nu_{1}$ is absolutely continuous with respect to $\nu_{0}+\nu_{2}$;

(iii) if $\alpha=d \nu_{0} / d\left(\nu_{0}+\nu_{2}\right), \beta=d \nu_{1} / d\left(\nu_{0}+\nu_{2}\right)$, and $\gamma=d \nu_{2} / d\left(\nu_{0}+\nu_{2}\right)$, then $\beta^{2}-4 \alpha y=0$ a.e. $\left(\nu_{0}+\nu_{2}\right)$.

In summary then, we have shown that if $B(\phi, \psi)$ is the covariance of a process with independent values depending only on $\phi, \psi$ and their first derivatives (in the sense of equation $\left(1^{\prime}\right)$ ), then we can write

$$
B(\phi, \phi)=\int\left(\phi^{2} \alpha+\phi \phi^{\prime} \beta+\phi^{\prime 2} \gamma\right) d \sigma
$$

for some positive Radon measure $\sigma$ and some locally $\sigma$-integrable functions $\alpha, \beta, \gamma$ with $\alpha \geq 0, \gamma \geq 0$ and $\beta^{2}-4 \alpha \gamma \leq 0$ a.e. ( $\left.\sigma\right)$.

Of course, the questions discussed above are quite general ones about inner products involving functions and their derivatives and these results would appear to be useful in other contexts (see, for example, Helton [1972]). I conjecture the following generalization of Theorem 1:

If $Q(\phi)=\Sigma_{i, j=0}^{M} \int \phi^{(i)} \phi^{(j)} d \mu_{i j}$ is a positive quadratic form on $\mathcal{D}(\mathbf{R})$, then the measures $\mu_{i j}$ can be assumed to satisfy $\Sigma_{i, j=0}^{M} \int f_{i} f_{j} d \mu_{i j} \geq 0$ whenever $f_{0}, \ldots, f_{M} \in \mathscr{T}^{0}(\mathbf{R})$.

These results appeared in the author's doctoral dissertation written at M.I.T. under Professor R. M. Dudley who suggested the problem treated here.

\section{REFERENCES}

1. X. Fernique [1967], Processus linéaires, processus généralisés, Ann. Inst. Fourier (Grenoble) 17, fasc. 1, 1-92. MR 36 \#628.

2. I. M. Gel' fand and N. Ja. Vilenkin [1964], Generalized functions. Vol. 4: Some applications of harmonic analysis, Fizmatgiz, Moscow, 1961; English transl., Academic Press, New York and London. MR 26\#4173; 30 \#152.

3. J. W. Helton [1972], Jordan operators in infinite dimensions and Sturm-Liouville conjugate point theory, Bull. Amer. Math. Soc. 78, 57-61. MR 44 \#3143.

4. I. Namioka [1957], Partially ordered linear opological spaces, Mem. Amer. Math. Soc. No. 24. MR $20 \# 1193$.

DEPARTMENT OF MATHEMATICS, UNIVERSITY OF RHODE ISLAND, KINGSTON, RHODE ISL AND U2881 